\title{
Efetividade de proposta de capacitação para professores da rede pública e privada
}

\section{sobre avulsão dentária}

\author{
Effectiveness of training proposal for public and private teachers on dental avulsion \\ Efectividad de propuesta formativa para docentes públicos y privados sobre avulsión dental
}

\author{
Ana Cláudia Amorim Gomes \\ ORCID: https://orcid.org/0000-0003-0934-6086 \\ Universidade de Pernambuco, Brasil \\ E-mail: anacagomes@upe.br \\ Renata Kelly Nogueira Trajano \\ ORCID: https://orcid.org/0000-0002-3571-1713 \\ Universidade de Pernambuco, Brasil \\ E-mail: renata.trajano@upe.br \\ Gabriela Almeida Souza Leão Simonton \\ ORCID: https://orcid.org/0000-0002-5405-3402 \\ Universidade de Pernambuco, Brasil \\ E-mail: almeida.gabriela@live.com \\ Mariana Pereira de Souza da Silva \\ ORCID: https://orcid.org/0000-0002-1665-2965 \\ Universidade de Pernambuco, Brasil \\ E-mail: mariperass@gmail.com \\ Pamella Robertha Rosselinne Paixão Celerino \\ ORCID: https://orcid.org/0000-0002-4607-6161 \\ Universidade de Pernambuco, Brasi \\ E-mail: pamellapaixao25@gmail.com \\ Ana Beatriz Leme de Andrade \\ ORCID: https://orcid.org/0000-0003-0357-8417 \\ Universidade de Pernambuco, Brasil \\ E-mail: anabeatrizleme@hotmail.com \\ Adriane Tenório Dourado \\ ORCID: https://orcid.org/0000-0003-4659-0117 \\ Universidade de Pernambuco, Brasil \\ E-mail: adrianedourado@gmail.com
}

\begin{abstract}
Resumo
A avulsão dentária é o deslocamento do dente do seu alvéolo. Tem maior prevalência entre 7 a 12 anos, sendo o reimplante dentário a melhor conduta para um bom prognóstico. $\mathrm{O}$ manejo adequado dos professores nas escolas tem extrema importância, entretanto, em geral, não é executado devidamente por carência de conhecimento. Objetivo: Avaliar o nível de conhecimento, prévio e posterior a capacitação, de professores da rede pública e privada, acerca do correto manejo da avulsão dentária. Material e Métodos: Ensaio comunitário com educadores de escolas públicas e privadas. Aplicou-se um formulário a 64 professores de cada rede e, em seguida, realizou-se a capacitação dos mesmos. Após um mês, houve a reavaliação dos professores já capacitados. Os dados foram digitados na planilha EXCEL e o programa para obtenção dos cálculos estatísticos foi o SPSS na versão 23. Resultados: Apenas 3,1\% dos professores de escolas públicas e $15,6 \%$ da rede privada já tinham recebido orientações sobre traumatismo dento-alveolar. Na primeira avaliação, a questão para onde levar o aluno com dente avulsionado mostrou diferença significativa entre os dois grupos de educadores. Já sobre o melhor meio para armazenar o dente, apenas 5,5\% escolheu o "leite", mostrando conhecimento insuficiente sobre o assunto. Após a capacitação, evidenciou-se aumento do conhecimento e segurança para um correto manejo, onde $51,6 \%$ passaram a se sentir capazes a realizar o reimplante dentário. Conclusão: Verificou-se conhecimento insuficiente, principalmente na rede pública. Entretanto, houve melhora dos índices após a capacitação, evidenciando a importância do trabalho multidisciplinar entre cirurgiões-dentistas e educadores.
\end{abstract}

Palavras-chave: Avulsão dentária; Reimplante dentário; Comunicação interdisciplinar.

\section{Abstract}

Dental avulsion is the displacement of the tooth from its alveolus. Has a higher prevalence between 7 and 12 years. Dental replantation is the best approach for a good prognosis. Adequate management of teachers in schools is extremely important, however, in general, they do not perform properly due to lack of knowledge. Objective: Assess the level of knowledge, before and after training, of teachers from public and private schools about the correct management of 
dental avulsion. Material and Methods: This community-typed study was conducted with educators from public and private schools networs. A questionnaire was applied to 64 teachers from each network and, afterwards, their training was carried out. After a month, there was a reassessment of teachers already trained. The data were entered into the EXCEL spreadsheet and the program used to obtain statistical calculations was SPSS in version 23. Results: Only $3.1 \%$ of public school teachers and $15.6 \%$ of the private network had already received guidance on dentoalveolar trauma. In the first assessment, the question of where to take the student with an avulsed tooth showed a significant difference between the two groups of educators. Regarding the best way to store the tooth, only 5.5\% chose "milk", showing insufficient knowledge on the subject. After training, there was an increase in knowledge and safety for correct management, where 51.6\% started to feel able to perform dental replantation. Conclusion: There was insufficient knowledge, mainly in the public network. However, there was an improvement in the rates after training, showing the importance of multidisciplinary work between dentists and educators.

Keywords: Tooth avulsion; Dental replantation; Interdisciplinary communication.

\section{Resumen}

La avulsión del diente es el desplazamiento del diente de su alvéolo. Tiene una mayor prevalencia entre los 7 y los 12 años, siendo la reimplantación dental el mejor enfoque para un buen pronóstico. La adecuada gestión de los docentes en las escuelas es de suma importancia, sin embargo, en general, no se ejecuta adecuadamente por falta de conocimiento. Objetivo: Evaluar el nivel de conocimientos, antes y después de la formación, de docentes públicos y privados, sobre el correcto manejo de la avulsión dental. Material y métodos: Ensayo comunitario con educadores de colegios públicos y privados. Se aplicó un formulario a 64 docentes de cada red y, posteriormente, se realizó su formación. Después de un mes, hubo una reevaluación de los maestros ya capacitados. Los datos se ingresaron en la hoja de cálculo EXCEL y el programa para la obtención de cálculos estadísticos fue el SPSS en versión 23. Resultados: Solo el 3.1\% de los docentes de escuelas públicas y el $15.6 \%$ de la red privada ya habían recibido orientación en trauma dental alveolar. En la primera evaluación, la pregunta de dónde llevar al estudiante con un diente avulsionado mostró una diferencia significativa entre los dos grupos de educadores. En cuanto a la mejor forma de almacenar el diente, solo el 5,5\% eligió "leche", mostrando un conocimiento insuficiente sobre el tema. Después de la capacitación, hubo un aumento en el conocimiento y la seguridad para un correcto manejo, donde el 51,6\% comenzó a sentirse capaz de realizar la reimplantación dental. Conclusión: No hubo conocimiento suficiente, principalmente en la red pública. Sin embargo, hubo una mejora en las tasas después de la formación, mostrando la importancia del trabajo multidisciplinar entre odontólogos y educadores.

Palabras-clave: Avulsión dental; Reimplantación dental; Comunicación interdisciplinar.

\section{Introdução}

O traumatismo dentário é um dos problemas de saúde bucal de maior relevância na infância, onde as consequências podem variar desde uma simples fratura até uma avulsão dentária (Costa et al., 2019; Gomes, Bittencourt, Martins, Paiva \& Bendo, 2020). A avulsão dentária é definida como o completo deslocamento do dente do seu alvéolo de origem, ocasionando a ruptura do feixe vasculonervoso apical e das fibras do ligamento periodontal. Dentre as lesões por traumatismo dentário, a avulsão possui uma prevalência significativa entre 0.5-16\% (Fouad et al., 2020; Rodrigues, Rodrigues \& Rocha, 2017; Menegotto, Scatena, Pereira, Werle \& Oliveira, 2017).

Os dentes mais acometidos são os incisivos superiores, devido a sua posição na arcada dental, especialmente em dentições jovens, com alta prevalência na faixa etária de 7 a 12 anos (Guedes-Pinto, Bonecker, Rodrigues \& Crivello, 2009; Andreasen \& Andreasen, 2011). Este fato se justifica devido ao desenvolvimento radicular incompleto e ao periodonto ser pouco resiliente. Além disso, é na fase escolar que acidentes como quedas associadas a brincadeiras e prática de esportes são mais comuns, constituindo a principal causa de lesões traumáticas dentárias (Menegotto et al., 2017; Alsadhan, Alsavari \& Abuabat, 2018).

Este tipo de trauma, além das lesões físicas, tem grande impacto no aspecto emocional por afetar diretamente a qualidade de vida dos indivíduos, podendo gerar complicações nas relações sociais. Dessa forma, as ações tomadas no local do acidente são extremamente importantes (Fouad et al., 2020; Servat, Schistel \& Massignan, 2019). O reimplante dentário é considerado a melhor forma de tratamento na maioria dos casos, porém quando não há condições de realizá-lo, deve-se armazenar o dente em um meio adequado, e procurar por atendimento odontológico breve, com o intuito de manter a viabilidade celular 
(Fouad et al., 2020; Guedes-Pinto et al., 2009; Menegotto et al., 2017; Lubaszewski, Raldi, Pinto, \& Habitante, 2015; Scandiuzzi, Sampaio, Moreira, Amaral \& Murrer, 2018).

A fim de aumentar a probabilidade de sucesso, é necessário que o reimplante seja feito de forma imediata e apropriada (Neto, Souza, Freire, Silva, Medeiros \& Cavalcanti, 2012), os primeiros cuidados geralmente são fornecidos por profissionais que não são da área da saúde e que se encontram no local do acidente. Devido ao fato de ocorrerem com grande frequência no ambiente escolar, a participação efetiva dos professores diante de uma avulsão dentária tem extrema importância, uma vez que irá afetar diretamente o prognóstico a longo prazo do dente avulsionado. Contudo, devido a uma carência de conhecimento, o pronto atendimento não é realizado da forma correta (Scandiuzzi et al., 2018).

Neste contexto, o objetivo desse estudo foi verificar a efetividade da capacitação sobre avulsão dental com professores de ensino infantil e fundamental de escolas públicas e privadas, comparando o nível de conhecimento prévio e posterior a capacitação.

\section{Metodologia}

Trata-se de um estudo de intervenção do tipo ensaio comunitário, que consiste na implementação ou avaliação de intervenções dirigidas antes e após a prevenção primária, através da modificação de fatores de risco em uma população bem definida. Nessa pesquisa, a capacitação dos professores representa a intervenção (Nedel \& Silveira, 2016; Capp \& Nienov, 2020).

A população alvo foi constituída de 64 professores de ensino infantil e fundamental de seis escolas públicas e oito escolas privadas da região metropolitana do Recife-PE. Foi realizada, presencialmente, uma entrevista por meio de formulário e, logo em seguida, houve uma capacitação com os grupos de professores de cada escola. As orientações foram passadas por meio de aula expositiva de duração média de 25 minutos, com apresentação de slides, e entrega de folheto autoexplicativo e ilustrado sobre os primeiros socorros em casos de avulsão dental. Passado o período de um mês da capacitação, foi realizada uma nova entrevista, utilizando o mesmo formulário, dirigida aos mesmos professores já capacitados, avaliando desta forma, a validação da capacitação.

De acordo com a resolução 466/12 do Conselho Nacional de Saúde, relativa à pesquisa em seres humanos, foi elaborado um Termo de Consentimento Livre e Esclarecido (TCLE) constando todas as informações apresentadas aos participantes sobre a pesquisa, sendo devidamente preenchido e assinado por cada professor no momento da aplicação do formulário. A pesquisa devidamente submetida e aprovada pelo Comitê de Ética em Pesquisa pelo número de parecer 1.013.565 e número CAAE 39192414.6.0000.5207.

A coleta de dados ocorreu através da observação direta intensiva, cujo objetivo principal foi obter informações dos entrevistados sobre o tema estabelecido na pesquisa. Quanto ao tipo de entrevista, foi empregada a padronizada ou estruturada, em que o autor foi o entrevistador e o mesmo utilizou um formulário validado (Ludgero et al., 2012). O roteiro foi previamente estabelecido, portanto, as perguntas foram pré-determinadas. O objetivo da padronização foi obter dos entrevistados respostas às mesmas perguntas, permitindo a comparação com o mesmo conjunto de perguntas, de forma que as diferenças ocorressem entre os respondentes e não entre as perguntas.

Os dados foram analisados e para avaliar a diferença entre os dois estudos, com os dois tipos de rede escolar (pública e privada) foi utilizado o teste Qui-quadrado de Pearson. A margem de erro utilizada foi de 5\%. Os dados foram digitados na planilha EXCEL e posteriormente exportados e analisados no software SPSS na versão 23.

\section{Resultados}

As tabelas 1 e 2 apresentam os resultados da pesquisa referente à avaliação prévia a palestra, de acordo com o tipo de escola. Através dos resultados apresentados na Tabela 1, pode-se observar a predominância de profissionais do sexo feminino 
nas escolas pesquisadas, sendo que cada tipo de escola apresentou somente 01 profissional do sexo masculino como participante da pesquisa. A maioria encontra-se na faixa etária 35 a 44 anos, correspondendo a 42,2\% entre os pesquisados de escolas particulares e $39,1 \%$ entre os profissionais de escolas públicas. Em menor proporção, o percentual de professores com 45 anos ou mais variou entre $29,7 \%$ a $35,9 \%$ dos entrevistados, sendo que um percentual ainda menor foi obtido quando comparada a faixa etária 19 a 34 anos de cada grupo, apresentando variações de 25 a $28,1 \%$.

O tempo de ensino de 15 anos ou mais predominou na pesquisa, sendo encontrado em 42,2\% dos professores de escolas particulares e 45,3\% de escolas públicas. Os demais resultados relacionados com o tempo de ensino mostraram em rede particular 6 a 10 anos (29,7\%), 1 a 5 anos (18,8\%), enquanto que o grupo pesquisado nas escolas públicas, seguiu-se em faixa de tempo de ensino 11 a 15 anos (23,4\%) e 6 a 10 anos (20,3\%). Quando questionados acerca de conhecimentos relacionados ao tratamento de trauma dento alveolar, pequena quantidade da amostra apresentou resposta positiva, sendo a maioria dos casos em escolas particulares $(15,6 \%)$, nas escolas públicas apenas 3,1\% possuíam algum conhecimento. Segundo o questionamento acerca de haver presenciado algum acidente em que houve total deslocamento do dente para fora da boca poucas foram as respostas afirmativas, sendo que mais uma vez as escolas particulares apresentaram percentual maior nestes relatos $(26,6 \% \mathrm{x}$ 17,2\%). Considerando margem de erro fixada (5\%), registrou-se significativa diferença $(\mathrm{p}<0,05)$ entre os grupos avaliados nas variáveis: tempo de ensino e na questão “Já teve orientações sobre o tratamento de trauma dento alveolar?”.

Tabela 1 - Informações pessoais e profissionais na avaliação antes segundo o tipo de escola.

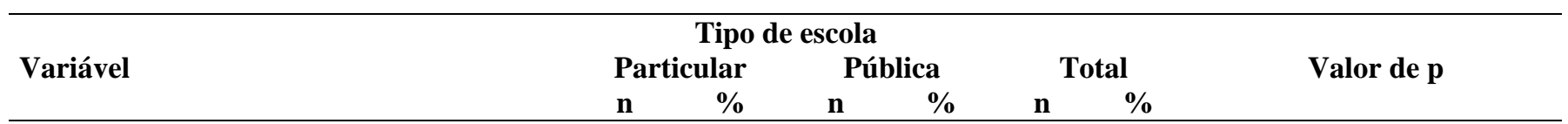

\section{Total}

Sexo

Masculino

Feminino

Faixa etária (anos)

19 a 34

35 a 44

45 ou mais

64

\section{Tempo de ensino (anos)}

Menos de 1

1 a 5

6 a 10

11 a 15

Mais de mais

Teve orientações sobre o tratamento trauma dento alveolar

Sim

Não

$\begin{array}{llllll}64 & 100,0 & 64 & 100,0 & 128 & 100,0\end{array}$

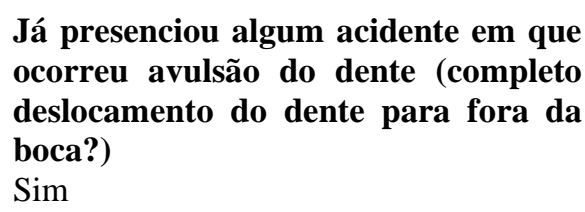

Sim

$\begin{array}{cccccc}1 & 1,6 & 1 & 1,6 & 2 & 1,6 \\ 63 & 98,4 & 63 & 98,4 & 126 & 98,4 \\ & & & & & \\ 18 & 28,1 & 16 & 25,0 & 34 & 26,6 \\ 27 & 42,2 & 25 & 39,1 & 52 & 40,6 \\ 19 & 29,7 & 23 & 35,9 & 42 & 32,8\end{array}$

(*) Associação significativa a 5\% $\mathrm{p}^{(2)}=0,015^{*}$

$\begin{array}{cccccc}10 & 15,6 & 2 & 3,1 & 12 & 9,4 \\ 54 & 84,4 & 62 & 96,9 & 116 & 90,6\end{array}$

$$
\mathrm{p}^{2}=0,015^{*}
$$

$\begin{array}{ccccccc} & & & & & & \mathrm{p}^{(1)}=0,042^{*} \\ 1 & 1,6 & 2 & 3,1 & 3 & 2,3 & \\ 12 & 18,8 & 5 & 7,8 & 17 & 13,3 & \\ 19 & 29,7 & 13 & 20,3 & 32 & 25,0 & \\ 5 & 7,8 & 15 & 23,4 & 20 & 15,6 & \\ 27 & 42,2 & 29 & 45,3 & 56 & 43,8 & \end{array}$

$$
\mathrm{p}^{(1)}=1,000
$$

$$
\mathrm{p}^{(2)}=0,750
$$

$$
\mathrm{p}^{(2)}=0,200
$$

$\begin{array}{cccccc}17 & 26,6 & 11 & 17,2 & 28 & 21,9 \\ 47 & 73,4 & 53 & 82,8 & 100 & 78,1\end{array}$


(1) Pelo teste Exato de Fisher

(2) Pelo teste Qui-quadrado de Pearson.

Fonte: Autores.

Em relação às questões sobre o conhecimento em avulsão na avaliação prévia à capacitação (Tabela 2) ressalta-se que na questão P1, "durante um jogo de futebol um aluno de 10 anos de idade tem seu incisivo central (dente da frente avulsionado (deslocado completamente para fora da boca)", a maioria dos entrevistados em ambos os tipos de escola respondeu se tratar de um dente permanente, com 70,3\% entre os participantes das escolas particulares e 62,5\% entre os pesquisados da rede pública, enquanto que os demais responderam tratar-se de um dente decíduo ou não ter certeza, com taxas variando de $12,5 \%$ a $25,0 \%$.

Na questão P2, “supondo que o dente tenha caído no chão, o que faria?", todos os profissionais da amostra de escolas particulares $(100 \%)$ responderam que pegariam o dente, enquanto que $85,9 \%$ da amostra das escolas públicas informou tal resposta. Na questão P3, “caso você tenha pegado o dente o que faria?", predominou a resposta informando que limparia com água ou algum líquido, com percentual variando de 60,0\% a 60,9\% sendo que o segundo maior percentual na rede particular correspondeu aos que não limpariam o dente $(34,4 \%)$, enquanto que no grupo da rede pública o segundo maior percentual $(25,5 \%)$ correspondeu a resposta "limparia com um pano de papel".

Na questão P4, "se um aluno lhe procurasse com um dente avulsionado (que saiu da boca) na mão para onde você o levaria?", 60,9\% da amostra das escolas particulares respondeu que levaria ao dentista, enquanto que nas outras três categorias os percentuais que variaram de $6,3 \%$ a $18,7 \%$. Variações foram relatadas na resposta do grupo das escolas públicas, com percentuais das 4 categorias variando de 17,2\% a 34,4\%. Quanto a questão P5, "quando se deve procurar ajuda profissional numa situação em que um dente permanente é avulsionado?”, a maioria em cada tipo de escola respondeu imediatamente sendo que os percentuais das escolas particular e pública variaram de $82,8 \%$ a $87,5 \%$.

Na questão P6, "seria capaz de colocar de volta (reimplantar) um dente em seu alvéolo (no lugar de origem)?”, a maior parte dos entrevistados respondeu negativamente à questão com $81,2 \%$ no grupo das escolas particulares e $89,1 \%$ no grupo das escolas públicas. Na questão P7, "se não decidisse reimplantar (colocar de volta) o dente, qual meio você utilizaria para armazenar?", 43,8\% dos participantes da rede particular informaram armazenar em água, seguido da resposta soro fisiológico (26,6\%), enquanto que no grupo da rede pública as respostas mais frequentes foram: soro fisiológico (39,1\%), água (37,5\%) e álcool (20,3\%). Diferenças significativas entre os dois grupos ( $<<0,05)$ ocorreram nas questões P2, P3, P4 e P7. 
Tabela 2 - Avaliação das questões sobre o conhecimento sobre avulsão na avaliação antes segundo o tipo de escola.

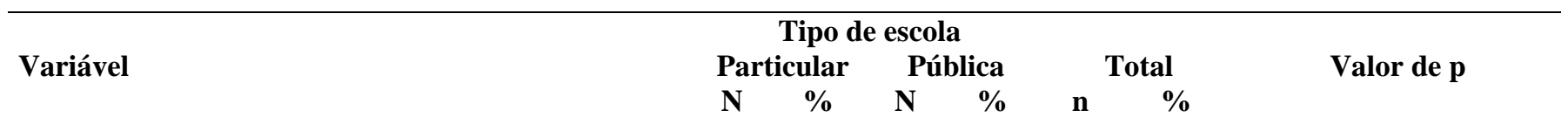

\section{Total}

$64 \quad 100,0 \quad 64 \quad 100,0 \quad 128 \quad 100,0$

P1. Durante um jogo de futebol um aluno de 10 anos de idade tem seu incisivo central (dente da frente) avulsionado (deslocado completamente para fora da boca. $O$ dente avulsionado é geralmente um dente permanente ou decíduo (leite)?

Permanente

Decíduo

Não tenho certeza

P2. Supondo que o dente tenha caído no chão, o que faria?

Pegaria o dente

Não pegaria o dente

P3. Caso você tenha pego o dente. O que faria?

Limparia com água ou algum líquido

Limparia com um pano ou papel

Não limparia o dente

P4. Se um aluno lhe procurasse com um dente avulsionado (que saiu da boca) na mão. Para onde você o levaria?

Assistência médica

Dentista

Casa do aluno

Outro lugar

P5. Quando se deve procurar ajuda profissional numa situação em que um dente permanente é avulsionado?

Imediatamente

Dentro de 30 minutos

Em poucas horas

P6. Seria capaz de colocar de volta (reimplantar) um dente em seu alvéolo (no lugar de origem)?

Sim

Não

$$
\mathrm{p}^{(1)}=0,290
$$

$\begin{array}{llllll}45 & 70,3 & 40 & 62,5 & 85 & 66,4\end{array}$

$\begin{array}{llllll}10 & 15,6 & 8 & 12,5 & 18 & 14,1\end{array}$

$\begin{array}{llllll}9 & 14,1 & 16 & 25,0 & 25 & 19,5\end{array}$

$\begin{array}{llllll}64 & 100,0 & 55 & 85,9 & 119 & 93,0\end{array}$

$\mathrm{p}^{(2)}=0,003 *$

$\begin{array}{llllll}- & - & 9 & 14,1 & 9 & 7,0\end{array}$

$\begin{array}{llllll}39 & 60,9 & 33 & 60,0 & 72 & 60,5\end{array}$

$\begin{array}{llllll}3 & 4,7 & 14 & 25,5 & 17 & 14,3\end{array}$

$\begin{array}{llllll}22 & 34,4 & 8 & 14,5 & 30 & 25,2\end{array}$

$\mathrm{p}^{(1)}=0,001^{*}$

$\begin{array}{cccccc}9 & 14,1 & 11 & 17,2 & 20 & 15,6 \\ 39 & 60,9 & 18 & 28,1 & 57 & 44,5 \\ 4 & 6,3 & 13 & 20,3 & 17 & 13,3 \\ 12 & 18,7 & 22 & 34,4 & 34 & 26,6\end{array}$

$\mathrm{p}^{(2)}=0,800$

$\begin{array}{llllll}56 & 87,5 & 53 & 82,8 & 109 & 85,2\end{array}$

$\begin{array}{llllll}1 & 1,6 & 1 & 1,6 & 2 & 1,6\end{array}$

$\begin{array}{llllll}7 & 10,9 & 10 & 15,6 & 17 & 13,3\end{array}$

P7. Se não decidisse reimplantar (colocar de volta) o dente, qual meio você usaria para armazenar?

Água

Leite

Saliva

Soro fisiológico

Suco de frutas

Álcool

(*) Associação significativa a 5\%

(1) Pelo teste Qui-quadrado de Pearson

(2) Pelo teste Exato de Fisher.

Fonte: Autores. 
As Tabelas 3 e 4 apresentam os resultados da pesquisa referente à avaliação posteior a palestra, de acordo com o tipo de escola. Através dos resultados apresentados na Tabela 3, verifica-se que na avaliação após capacitação, a amostra permanece composta por maioria do sexo feminino, com 01 profissional do sexo masculino em cada tipo de escola pesquisada. A faixa etária de 35 a 44 anos foi mais prevalente com 42,2\% entre os pesquisados da escola particular e 40,6\% entre os participantes da escola pública e os percentuais da faixa 45 ou mais variaram de $29,7 \%$ a $35,9 \%$ e a faixa 19 a 34 anos de $23,4 \%$ a $28,1 \%$. O tempo de ensino de 15 anos ou mais também persistiu como mais frequente em cada grupo pesquisado, sendo 42,2\% entre os pesquisados da rede particular e $45,3 \%$ na rede pública.

Quando questionados se já receberam orientações sobre o tratamento de trauma dento alveolar, a maior parte respondeu afirmativamente com percentuais variando de 92,2\% a 93,8\%; a minoria em cada grupo ou tipo de escola respondeu positivamente ao questionamento relacionado com o fato de já ter presenciado algum acidente em que ocorreu avulsão do dente, sendo que a maior parte das respostas positivas foram verificadas no grupo das escolas particulares em relação as escolas públicas $(25,0 \% \times 14,1 \%)$. Faixa de tempo de ensino foi a única variável com diferença significativa $(\mathrm{p}<0,05)$ entre os dois grupos.

Tabela 3 - Informações pessoais e profissionais na avaliação depois segundo o tipo de escola.

\begin{tabular}{|c|c|c|c|c|c|c|c|}
\hline \multirow{3}{*}{ Variável } & \multicolumn{4}{|c|}{ Tipo de escola } & & & \multirow{3}{*}{ Valor de p } \\
\hline & \multicolumn{2}{|c|}{ Particular } & \multicolumn{2}{|c|}{ Pública } & \multicolumn{2}{|c|}{ Total } & \\
\hline & $\mathbf{n}$ & $\%$ & $\mathbf{n}$ & $\%$ & n & $\%$ & \\
\hline Total & 64 & 100,0 & 64 & 100,0 & 128 & 100,0 & \\
\hline Sexo & & & & & & & $\mathrm{p}^{(1)}=1,000$ \\
\hline Masculino & 1 & 1,6 & 1 & 1,6 & 2 & 1,6 & \\
\hline Feminino & 63 & 98,4 & 63 & 98,4 & 126 & 98,4 & \\
\hline Faixa etária & & & & & & & $\mathrm{p}^{(2)}=0,714$ \\
\hline 19 a 34 & 18 & 28,1 & 15 & 23,4 & 33 & 25,8 & \\
\hline 35 a 44 & 27 & 42,2 & 26 & 40,6 & 53 & 41,4 & \\
\hline 45 ou mais & 19 & 29,7 & 23 & 35,9 & 42 & 32,8 & \\
\hline Tempo de ensino (anos) & & & & & & & $\mathrm{p}^{(1)}=0,042 *$ \\
\hline Menos de 1 & 1 & 1,6 & 2 & 3,1 & 3 & 2,3 & \\
\hline 1 a 5 & 12 & 18,8 & 5 & 7,8 & 17 & 13,3 & \\
\hline 6 a 10 & 19 & 29,7 & 13 & 20,3 & 32 & 25,0 & \\
\hline 11 a 15 & 5 & 7,8 & 15 & 23,4 & 20 & 15,6 & \\
\hline Mais de 15 & 27 & 42,2 & 29 & 45,3 & 56 & 43,8 & \\
\hline $\begin{array}{l}\text { Teve orientações sobre o tratamento } \\
\text { trauma dento alveolar }\end{array}$ & & & & & & & $\mathrm{p}^{(1)}=1,000$ \\
\hline $\operatorname{Sim}$ & 59 & 92,2 & 60 & 93,8 & 119 & 93,0 & \\
\hline Não & 5 & 7,8 & 4 & 6,3 & 9 & 7,0 & \\
\hline $\begin{array}{l}\text { Já presenciou algum acidente em que } \\
\text { ocorreu avulsão do dente (completo } \\
\text { deslocamento do dente para fora da } \\
\text { boca?) }\end{array}$ & & & & & & & $\mathrm{p}^{(2)}=0,119$ \\
\hline $\operatorname{Sim}$ & 16 & 25,0 & 9 & 14,1 & 25 & 19,5 & \\
\hline Não & 48 & 75,0 & 55 & 85,9 & 103 & 80,5 & \\
\hline
\end{tabular}

(*) Associação significativa a 5\%

(1) Pelo teste Exato de Fisher

(2) Pelo teste Qui-quadrado de Pearson.

Fonte: Autores. 
Quanto às questões relacionadas ao conhecimento sobre avulsão na avaliação após a capacitação (Tabela 4) verifica-se que: na questão P1, “durante um jogo de futebol um aluno de 10 anos de idade tem seu incisivo central (dente da frente) deslocado completamente para fora da boca", $85,9 \%$ entre os participantes da rede particular e 81,3\% entre os pesquisados da rede pública responderam ser um dente permanente, enquanto o restante da amostra respondeu se tratar de um dente decíduo ou não ter certeza, com variações de 4,7\% a 10,9\%. Na questão P2, “supondo que o dente tenha caído no chão, o que faria?”, um percentual de $100 \%$ da amostra das escolas particulares respondeu que pegaria o dente, enquanto 98,4\% dos profissionais da rede pública responderam afirmativamente este questionamento.

Na questão P3, “caso você tenha pegado o dente o que faria?", a maior parte dos entrevistados respondeu que limparia com água ou algum líquido, com valores que variaram de 79,7\% a 82,8\%, com o segundo maior percentual na rede particular correspondendo aos que não limpariam o dente, sendo 17,2\% na rede particular e 12,5\% na rede pública. Na questão P4, "se um aluno lhe procurasse com um dente avulsionado (que saiu da boca) na mão para onde você o levaria?”, a maioria em cada grupo respondeu que levaria ao dentista, com percentual de 79,7\% nas escolas particulares e 65,6\% nas escolas públicas, seguida da categoria assistência médica com percentuais que variaram de $14,1 \%$ a $18,8 \%$.

Quanto a questão P5, "quando se deve procurar ajuda profissional numa situação em que um dente permanente é avulsionado?", a maioria em cada tipo de escola respondeu imediatamente com valores de 96,9\% em cada grupo. Os percentuais dos que responderam sim na questão P6, "seria capaz de colocar de volta (reimplantar) um dente em seu alvéolo (no lugar de origem)?” variaram de 50,0\% a 53,1\%.

Na questão P7, “se não decidisse reimplantar (colocar de volta) o dente, qual meio você usaria para armazenar?”, a maioria respondeu leite, sendo 71,8\% no grupo das escolas particulares e 65,6\% na rede pública, nenhum participante citou suco de frutas e álcool e os percentuais das outras categorias variaram de 3,1\% a 18,8\%. Não foram registradas diferenças significativas entre os dois grupos $(\mathrm{p}<0,05)$ em nenhuma das questões avaliadas. 
Tabela 4 - Avaliação das questões sobre o conhecimento sobre avulsão na avaliação após segundo o tipo de escola.

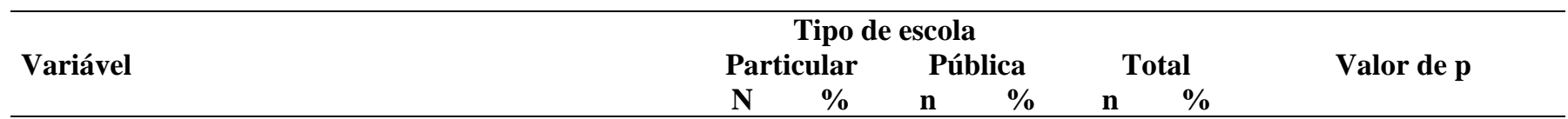

Total

$\begin{array}{llllll}64 & 100,0 & 64 & 100,0 & 128 & 0\end{array}$

P1. Durante um jogo de futebol um aluno de 10 anos de idade tem seu incisivo central (dente da frente) avulsionado (deslocado completamente para fora da boca). $O$ dente avulsionado é geralmente um dente permanente ou decíduo (leite)?

Permanente

Decíduo

Não tenho certeza

P2. Supondo que o dente tenha caído no chão, o que faria?

Pegaria o dente

Não pegaria o dente

P3. Caso você tenha pego o dente. O que faria?

Limparia com água ou algum líquido

Limparia com um pano ou papel

Não limparia o dente

P4. Se um aluno lhe procurasse com um dente avulsionado (que saiu da boca) na mão. Para onde você o levaria?

Assistência médica

Dentista

Casa do aluno

Outro lugar

P5. Quando se deve procurar ajuda profissional numa situação em que um dente permanente é avulsionado?

Imediatamente

Dentro de 30 minutos

Em poucas horas

P6. Seria capaz de colocar de volta (reimplantar) um dente em seu alvéolo (no lugar de origem)?

Sim

Não

P7. Se não decidisse reimplantar (colocar de volta) o dente, qual meio você usaria para armazenar?

Água

Leite

Saliva

Soro fisiológico

Suco de frutas

Álcool $\mathrm{p}^{(1)}=0,776$

$\begin{array}{cccccc}55 & 85,9 & 52 & 81,3 & 107 & 83,6 \\ 6 & 9,4 & 7 & 10,9 & 13 & 10,2 \\ 3 & 4,7 & 5 & 7,8 & 8 & 6,3\end{array}$

$\mathrm{p}^{(1)}=1,000$

$\begin{array}{llllll}64 & 100,0 & 63 & 98,4 & 127 & 99,2\end{array}$

$\begin{array}{llllll}51 & 79,7 & 53 & 82,8 & 104 & 81,3\end{array}$

$\mathrm{p}^{(1)}=0,758$

$\begin{array}{llllll}2 & 3,1 & 3 & 4,7 & 5 & 3,9\end{array}$

$\begin{array}{llllll}11 & 17,2 & 8 & 12,5 & 19 & 14,8\end{array}$

$\mathrm{p}^{(1)}=0,323$

$\begin{array}{llllll}9 & 14,1 & 12 & 18,8 & 21 & 16,4\end{array}$

$\begin{array}{llllll}51 & 79,7 & 42 & 65,6 & 93 & 72,7\end{array}$

$\begin{array}{llllll}2 & 3,1 & 5 & 7,8 & 7 & 5,5\end{array}$

$\begin{array}{llllll}2 & 3,1 & 5 & 7,8 & 7 & 5,5\end{array}$

$\mathrm{p}^{(1)}=1,000$

$\begin{array}{cccccc}62 & 96,6 & 62 & 96,9 & 124 & 96,9 \\ 1 & 1,6 & - & - & 1 & 0,8 \\ 1 & 1,6 & 2 & 3,1 & 3 & 2,3\end{array}$

$\begin{array}{llllll}32 & 50,0 & 34 & 53,1 & 66 & 51,6\end{array}$

$\begin{array}{llllll}32 & 50,0 & 30 & 46,9 & 62 & 48,4\end{array}$

$\mathrm{p}^{(2)}=0,724$

$\mathrm{p}^{(1)}=0,778$

(1) Pelo teste Exato de Fisher

(2) Pelo teste Qui-quadrado de Pearson.

Fonte: Autores. 


\section{Discussão}

Ao longo do desenvolvimento da pesquisa foram encontradas algumas dificuldades. A definição do tamanho da amostra para se atingir um número significativo de professores foi uma delas, devido a extensão da região estudada e a grande quantidade de escolas públicas e privadas existentes. Durante o contato com as escolas o maior obstáculo foi a falta de tempo oportuno no calendário escolar para realização da atividade como os professores. No momento da capacitação ou no momento da reaplicação do formulário, alguns professores estavam ausentes por motivos como: atestado, licença ou férias. Já no processo de preenchimento dos formulários alguns foram entregues incompletos, fazendo com que estes fossem excluídos.

Há uma carência de conhecimento dos professores, de escolas públicas e privadas, a respeito dos primeiros socorros a serem adotados caso presenciem uma avulsão dental. Os resultados obtidos no presente estudo corroboram com pesquisas já realizadas em todo o mundo (Sae-Lim \& Lim, 2001; Granville-Garcia, Lima, Santos \& Menezes, 2007; Curylofo, Lorenceitti \& Silva, 2012; Marcano-Caldera et al., 2018; Alsadhan et al., 2018).

O quantitativo de professores que afirmam ter recebido orientações sobre tratamento em casos de traumas dentoalveolares, antes da presente capacitação, é extremamente baixo em escolas privadas (15,6\%) e, principalmente, em escolas públicas $(3,1 \%)$. Esse fato reflete a deficiência de políticas de capacitação de professores sobre os acidentes que podem acontecer com as crianças em ambiente escolar e sobre como agir diante dessas situações. Em um estudo feito com educadores da rede particular de ensino, quando questionados se já vivenciaram alguma experiência em traumatismo dental, seja através de atendimento, palestra, leitura ou outros, apenas 16,7\% responderam “sim” (Alves, Freitas, Rosendo, Gominho \& Samento, 2016).

$\mathrm{Na}$ literatura, pode-se encontrar o modelo de protocolo frente a avulsão em dente permanente indicado pela Associação Internacional de Traumatologia Dentária (IADT) (Fouad et al., 2020). Esse protocolo é aceito e se repete em vários estudos, todavia, o desconhecimento deste manejo pela categoria de professores é notável pelos resultados obtidos nessa pesquisa e como mostra a análise feita em Riyadh, Saudi Arabia, onde apenas 6,2\% dos professores sabiam que diante de uma avulsão em dente permanente o correto seria reimplantar o dente na posição correta (Alsadhan et al., 2018).

Diante de tal situação, além de ter conhecimento do protocolo, reconhecer um dente decíduo ou permanente é de fundamental importância, pois não é aconselhável o reimplante de um dente decíduo, pelo risco de infecção e lesão do germe do dente permanente (Costa et al., 2014). Dessa forma, é imprescindível que o professor receba orientação sobre como identificar o dente avulsionado. No presente estudo, antes da capacitação realizada com os professores, 29,7\% dos profissionais de escola privada e 37,5\% de escola pública não sabiam diferenciar um dente permanente de um decíduo, ou responderam erroneamente no formulário. Entretanto, após passarem pela orientação, 83,6\% dos educadores de ambas as redes responderam corretamente.

Em um estudo feito em João Pessoa-PB com professores, quando questionados se guardariam o dente em caso de avulsão, observou-se que houve um aumento significativo de respostas afirmativas comparando-se o período antes e após palestra educativa, sugerindo uma adequada mudança de conduta destes profissionais (Trigueiro, Costa, Souza, Leilão \& Ortega, 2015). Assim, no presente estudo, mesmo antes da capacitação, todos os professores da rede particular já possuíam consciência da importância da recuperação do dente após a avulsão, quanto aos educadores da rede pública, 14,1\% não estavam cientes. Entretanto, após a capacitação, observou-se que apenas 1 profissional dos 128 entrevistados não respondeu o formulário corretamente, evidenciando uma importante mudança de conduta.

O procedimento de limpeza da superfície radicular também exerce influência no processo de cicatrização (Andreasen, Andreasen, Bakland \& Flores, 2000). Deste modo, quando questionados sobre o que fariam com o dente avulsionado, 60 a $60,9 \%$ dos professores respondeu que limparia com água ou algum líquido. Após o trabalho de orientação vemos um aumento significante na resposta correta, tanto da rede privada como da pública, atingindo os valores de $82,8 \%$ e $79,7 \%$, respectivamente. 
Corroborando com o estudo realizado no Kuwaiti, em que, quanto a limpeza de um dente avulsionado, verificou-se um aumento no nível de conhecimento de 5\% para 93\%, após a intervenção com os professores (Al-Asfour, Andersson \& Al-Jame, 2008).

No tocante ao local que se deve levar a criança após uma avulsão dental, houve uma diferença significativa entre o grupo dos educadores de escola pública e privada. No grupo da rede particular a maioria $(60,9 \%)$ respondeu que levaria ao dentista, já no grupo da escola pública somente $28,1 \%$ procederiam de tal forma. Esse fato pode ser decorrente da maior dificuldade para o acesso ao atendimento odontológico das crianças da rede pública pelo nível de condição socioeconômica. Concordando com o último percentual, em um estudo com professores de escola pública, apenas 7,1\% afirmaram que levariam a criança para atendimento odontológico (Menegotto et al., 2017).

Quando questionados sobre o momento que deveriam procurar ajuda profissional em uma situação de avulsão de dente permanente, a maioria, em cada tipo de escola (87,5\% - particular e 82,8\% - pública) e após orientações (em torno de 96\%), respondeu "imediatamente". Este fato se configura em algo positivo, uma vez que o reimplante idealmente deve ser feito no local do acidente e no menor tempo possível a fim de preservar a vitalidade das células sobre a raiz. A negligência ou a demora no tratamento do traumatismo dental afeta o prognóstico e a qualidade de vida dos pacientes, principalmente quando atinge os dentes anteriores (Mori, Turcio, Borro \& Mariusso, 2007; Tzighounalis \& Merglová, 2008; Baretta, Volpato, Galato \& Medeiros, 2017; Rodrigues et al.,2017; Servat et al., 2019; Neto et al., 2012).

Em estudos anteriores, uma parcela muito pequena de professores afirmou que seria capaz de reimplantar um dente avulsionado (Curylofo et al., 2012; Silva, Melo, Martins, Pavan \& Poi, 2012; Young, Wong \& Cheung, 2012), o que coincide com a primeira etapa dessa pesquisa, tanto em âmbito público $(18,8 \%)$ quanto privado $(10,9 \%)$, podendo ser entendido que a falta de conhecimento sobre o assunto reflete na insegurança para agir. Porém, na segunda etapa, 50,0\% dos professores de escola particular e 53,1\% de escola pùblica sentiram-se capazes de reimplantar o dente em seu alveolo de origem (Trigueiro et al.,2015; Lubaszewski et al., 2015).

Inúmeros estudos citam o leite como melhor meio de transporte para um dente avulsionado devido ao fácil acesso no momento do acidente, composição e osmolaridade compatíveis para a sobrevivência das células sobre a raiz por até 6 horas (Trigueiro et al.,2015; Rodrigues, Rodrigues \& Rocha, 2017; Scandiuzzi et al., 2018; Adnan, Lone, Khan, Hussain \& Nagi, 2018). Quanto aos participantes deste estudo, quando questionados antes da capacitação sobre qual meio utilizariam para transporte do dente, apenas 7,8\% da rede particular e 5,5\% da rede pública escolheram o leite. O mesmo se observa em pesquisa feita com professores do ensino intermediário do Kuwait, que também apresentaram porcentagem baixa em relação a tal conduta (3,2\%) (Al-Asfour, Andersson \& Al-Jame, 2008). Contudo, após a capacitação, este percentual aumentou consideravelmente, $71,8 \%$ e $68,8 \%$ para rede privada e pública, respectivamente.

Diante do exposto, fica evidente a relevância desse estudo ao abordar a necessidade da construção de programas educacionais multiprofissionais envolvendo cirurgiões-dentistas e professores, a respeito de traumatismo dento alveolar com ênfase na avulsão dental. Os professores, da rede pública ou privada, são profissionais que devem estar preparados a conduzir um trauma desse tipo. As palestras educativas, juntamente com a distribuição de folhetos explicativos sobre o tema, se mostram importantes ferramentas para contribuir com o aumento do nível de conhecimento, refletindo no manejo mais adequado diante da avulsão dental.

\section{Considerações Finais}

Por meio desta pesquisa, constatou-se uma deficiência de conhecimento dos professores, principalmente das escolas públicas, acerca da conduta adequada diante da avulsão dental. Entretanto, percebe-se uma melhora considerável dos índices 
após a capacitação, elevando o nível de conhecimento e de segurança da conduta perante estes casos entre os educadores da rede pública e privada, refletindo a efetividade da proposta de capacitação dos professores.

\section{Referências}

Adnan, S., Lone, M. M., Khan, F. R., Hussain, S. M., \& Nagi, S. E. (2018). Which is the most recommended medium for the storage and transport of avulsed teeth? A systematic review. Dent Traumatol, 34(2):59-70.

Alsadhan, S. A., Alsayari, N. F., \& Abuabat, M. F. (2018). Teachers' knowledge concerning dental trauma and its management in primary schools in Riyadh, Saudi Arabia. Int Dent J, 68(5):306-13.

Al-Asfour, A., Andersson, L., Al-Jame, Q. (2008). School teachers' knowledge of tooth avulsion and dental first aid before and after receiving information about avulsed teeth and replantation. Dental Traumatology, 24(1):43-9.

Alves, L. S. B., Freitas, V. J. G., Rosendo, R. A., Gominho, L. F., \& Sarmento, T. C. (2016). Avaliação do conhecimento de professores do ensino fundamental da rede particular sobre atendimento imediato de vítima de traumatismo dental. Rev da Fac de Odontologia, UPF, 20(3).

Andreasen, J., \& Andreasen, F. (2011). Texto e atlas colorido de traumatismo dental (3a ed). Artmed Editora.

Andreasen, J. O., Andreasen, F. M., Bakland, L. K., \& Flores, M. T. (2000). Manual de traumatismos dentários. Artes Médicas.

Beretta, R. J., Volpato, M. E. S., Galato, D., \& Medeiros, G. H. F. (2017). Avulsão dental como resultado de trauma: o grau de conhecimento de profissionais socorristas. Rev da Fac de Odontologia UPF, 22(1).

Capp, E., \& Nienov, O. H. (2020). Bioestatística quantitativa aplicada.

Costa, S. C., Amaral, T. A. S., Miranda, D. K., Nogueira, J. S. E., Santos, Y. C. S., Nunes, A. S., Vilhema, A. T., Silva L. D., Souza, S. A., Santos, R. H. S., Oliveira, M. C. S., \& Freitas, J. K. C. (2019). Terapia Preventiva Pós Traumatismo Dental na Primeira Infância por Reabilitação Protética Funcional: Relato de Caso. Acervo Saúde, 8(26), 766

Costa, L. E. D., Queiroz, F. S., Nóbrega, C. B. C., Leite, M. S., Nóbrega, W. F. S., \& Almeida, E. R. (2014). Trauma dentário na infância: avaliação da conduta dos educadores de creches públicas de Patos-PB. Rev odontol UNESP, 43(6):402-8.

Curylofo, P. A., Lorenceitti, K. T., \& Silva, S. R. C. (2012). Avaliação do conhecimento de professores sobre avulsão dentária. Arquivos em Odontologia. 48(3):175-80.

Fouad, A. F., Abbott, P. V., Tsilingaridis, G., Cohenca, N., Lauridsen, E., Bourguignon, C., Flores, A. T., Hicks, M. L., Lenzi, A. R., Malmgren, B., Moule, A. J., \& Tsukiboshi, M. (2020). International Association of Dental Traumatology guidelines for the management of traumatic dental injuries: 2. Avulsion of permanent teeth. Dent Traumatol, 36(4):331-42.

Gomes, P. R., Bittencourt, J. M., Martins, L. P., Paiva, S. M., \& Bendo, C. B. (2020). Traumatismo dentário na dentição decídua e condição socioeconômica: uma revisão crítica da literatura. Arq Odontol, 56(08):1-10.

Guedes-Pinto, A. C., Bonecker, M. J. S, Rodrigues, C. R. M. D., \& Crivello Junior, O. (2009). Odontopediatria. Santos.

Granville-Garcia, A. F., Lima, E. M., Santos, P. G., \& Manezes, V. A. (2007). Avaliação do conhecimento dos professores de educação física de Caruaru-PE sobre avulsão- reimplante. Pesquisa Brasileira em Odontopediatria e Clínica Integrada, (1):15-20.

Lubaszewski, V. P. A., Raldi, D. P., Pinto, C. A., \& Habitante, S. M. (2015). Avaliação da conduta emergencial em casos de avulsão dentária antes e após palestras educativas. ClipeOdonto, 7(1):9-19..

Ludgero, L. A., Santos, T. S., Fernandes, A. V., Melo, D. G., Peixoto, A. C., Araújo, F. A. C., \& Dourado, A. T. (2012). Knowledge regarding emergency management of avulsed teeth among elementary school teachers in Jaboatão dos Guararapes, Pernambuco, Brazil. Indian J Dent Res, $23(5): 585$.

Marcano-Caldera, M., Mejía-Cardona, J. L., Parra Sanchez, J. H., Méndez, E. C., Covo, E. M., \& Sierra, G. V. (2018). Knowledge about emergency dental trauma management among school teachers in Colombia: A baseline study to develop an education strategy. Dent Traumatol, 34(3):164-74.

Menegotto, A., Scatena, C., Pereira, J. T., Werle, S. B., \& Oliveira, R. S. (2017). Avaliação do conhecimento dos professores de escolas públicas quanto ao manejo da avulsão dentária em crianças. Revista perspectiva: ciência e saúde, 2(1):83-94.

Mori G. G., Turcio K. H. L., Borro V. P. B., \& Mariusso Â. M. (2007). Evaluation of the knowledge of tooth avulsion of school professionals from Adamantina, São Paulo, Brazil: Knowledge of tooth avulsion of school professionals. Dental Traumatology, 23(1):2-5.

Nedel, W. L., \& Silveira, F. (2016). Different research designs and their characteristics in intensive care. Revista Brasileira de Terapia Intensiva, 28(3).

Neto, J. M. A. S., Souza, L. B., Freire, A. C. M., Silva, C. C. C., Medeiros, M. L. B. B., \& Cavalcanti, T. C. (2020). Diagnóstico e tratamento na avulsão dentária: uma revisão de literatura. Acervo Saúde, (40):e2657.

Rodrigues, T. L. C., Rodrigues, F. G., \& Rocha, J. F. (2017). Avulsão dentária: proposta de tratamento e revisão da literatura. RO_UNICID, $22(2): 147$.

Sae-Lim, V., \& Lim, L. P. (2001). Dental trauma management awareness of Singapore pre-school teachers. Dent Traumatol, 17(2):71-6.

Scandiuzzi. S., Sampaio, L. G. C., Moreira, M. B., Amaral, R. C., \& Murrer, R. D. (2018). Avaliação do conhecimento de professores do ensino fundamental 
Research, Society and Development, v. 10, n. 3, e19010313086, 2021

(CC BY 4.0) | ISSN 2525-3409 | DOI: http://dx.doi.org/10.33448/rsd-v10i3.13086

sobre avulsão e reimplante dentário. Rev Bras Odontol., 75.

Servat, R. L., Schistel, L. C., \& Massignan, C. (2019). Conhecimento de responsáveis sobre traumatismo dentário em crianças. RFO UPF, 24(2):220-8.

Silva, E., Melo, M., Martins, C., Pavan, A., \& Poi, W. (2012). Avulsão nas escolas: nível de conhecimento dos professores e educadores. Rev Odontol UNESP, 41:71.

Trigueiro, M., Costa, M. M. T. M. C., Souza, I. C. G., Leilão, G. L. N. C., \& Ortega, A. O. L. (2015). Avulsão dentária: efeito da informação na mudança de comportamento dos professores do ensino fundamental. ROBRAC, 24(69):57-61.

Tzigkounakis, V., \& Merglová, V. (2008). Attitude of Pilsen primary school teachers in dental traumas. Dental Traumatology, 24(5):528-31.

Young, C., Wong K., \& Cheung, L. K. (2012). Emergency management of dental trauma: knowledge of Hong Kong primary and secondary school teachers. Hong Kong Med J, (5):362-70. 